\title{
Une adénylyl cyclase sensible aux bicarbonates pour activer les spermatozoïdes?
}

L'activation des spermatozoïdes est un des processus indispensable à la fécondation. Chez les mammifères, les spermatozoïdes acquièrent leur potentiel de mobilité fonctionnelle pendant leur trajet dans l'épididyme, mais ce n'est qu'après leur dilution dans le liquide séminal, au moment de l'éjaculation, qu'ils deviennent réellement mobiles. L'acquisition de cette mobilité n'est cependant pas suffisante, et les spermatozoïdes doivent aussi subir une maturation, processus appelé capacitation, pendant laquelle leurs mouvements peuvent devenir "hyperactifs", avec de larges mouvements de tête orientés dans différentes directions, ce qui permettrait de favoriser leur rencontre avec... l'ovocyte [1]. Ces propriétés requièrent de l'AMPc intracellulaire et sont activées par les bicarbonates dont la concentration, de $5 \mathrm{mM}$ dans l'épididyme caudal avant l'éjaculation, augmente à $25 \mathrm{mM}$ quand le sperme se mélange au liquide séminal. Une équipe américaine vient de préciser les mécanismes de cette activation en montrant que les bicarbonates stimulent directement une adénylyl cyclase soluble exprimée principalement par les cellules germinales mâles [2].

Une activité soluble adénylate cyclase avait en effet été détectée, il y a plus de vingt ans, dans des extraits cytosoliques provenant de testicules de mammifères. Ces caractéristiques sont différentes de celles des enzymes de la superfamille des adénylate cyclases transmembranaires qui interagissent avec les protéines G (revue dans [3]) : sa taille est en effet plus petite et son activité est dépendante de la présence de $\mathrm{Mn}^{++}$et insensible aux protéines $\mathrm{G}$ ou à la forskoline. Ce n'est en fait que l'an dernier que la même équipe a pu cloner l'ADNc codant l'enzyme soluble, démontrant ainsi qu'il s'agit d'une forme moléculaire distincte des
L'ADNc code en fait pour une protéine de $187 \mathrm{kDa}$ dépourvue de segment transmembranaire, et dont la partie C-terminale ne présente aucune similitude avec des protéines connues, alors que la partie N-terminale possède deux domaines catalytiques C1 et C2. De façon surprenante, la protéine purifiée directement avait un poids moléculaire de $48 \mathrm{kDa}$ seulement et ne contenait vraisemblablement que les deux domaines catalytiques. L'hypothèse retenue a été celle d'un clivage protéolytique de la protéine de $187 \mathrm{kDa}$, l'étude en immunoblot révélant que la forme de $187 \mathrm{kDa}$ est prédominante dans les testicules, tandis que celle de $48 \mathrm{kDa}$ l'est dans le sperme. L'activité de cette adénylate cyclase, qu'elle soit utilisée sous sa forme purifiée à partir de testicules, ou recombinante, ou exprimée dans une lignée cellulaire, est stimulée par l'ajout de bicarbonates (sous forme de $\mathrm{NaHCO}_{3}$ ou $\mathrm{KHCO}_{3}$ ), et ceci de façon indépendante des modifications du $\mathrm{pH}$ intracellulaire [3]. Cette activation est aussi observée avec les ions bisulfites $\left(\mathrm{Na}_{2} \mathrm{SO}_{3}\right.$ ou $\left.\mathrm{NaHSO}_{3}\right)$ dont la structure est proche de celle des bicarbonates, mais pas avec les ions chlorides, sulfates ou phosphates. De plus, l'expression d'une protéine tronquée ne contenant que les deux domaines catalytiques et correspondant vraisemblablement à la protéine de $48 \mathrm{kDa}$ est suffisante pour permettre l'activation de l'enzyme. En revanche, une forme soluble purifiée de l'enzyme membranaire de type $\mathrm{V}$ n'est pas sensible aux bicarbonates. Il apparaît donc que cette caractéristique puisse être spécifique à l'enzyme soluble. Certaines cellules comme les spermatozoïdes pourraient donc posséder deux systèmes de transduction distincts impliquant l'AMPc, selon que l'adénylate cyclase est sous forme soluble ou membranaire. On ne sait cependant pas si la production d'AMPc par l'une ou l'autre de ces enzymes stimule, en aval, les mêmes voies ou des voies spécifiques.

Un des aspects les plus originaux de l'étude de cette adénylate cyclase se situe probablement au niveau phylogénétique. En effet, si l'homologie entre les deux domaines catalytiques C1 et C2 de l'enzyme soluble, ainsi que leur homologie avec ceux des formes membranaires, est faible, C1 et C2 sont en revanche l'un et l'autre très conservés avec certains domaines catalytiques des adénylates cyclases des cyanobactéries. Ceci suggère qu'au cours de l'évolution, la forme soluble dérive de la fusion de protéines bactériennes distinctes plutôt que de la duplication d'un unique domaine catalytique, et que les deux domaines C1 et C2 ont évolué indépendamment des domaines catalytiques des enzymes membranaires eucaryotes. En outre, la démonstration d'une stimulation de l'activité adénylate cyclase d'une cyanobactérie (Spirulina platensis) par les bicarbonates indique que ces enzymes pourraient jouer le rôle de directeur chimique, et que cette propriété est extrêmement bien conservée au cours de l'évolution.

1. Jouannet P, Serres C. Mouvement normal et pathologique du spermatozoïde humain. Med Sci $1995 ; 11: 555-62$.

2. Chen Y, Cann MJ, Livtin TN, et al. Soluble adenylyl cyclase as an evolutionarily conserved bicarbonate sensor. Science 2000 ; 289 : 625-8.

3. Hanoune J, Pouille Y, Tzavara E, et al. Adenylyl cyclases : structure, regulation and function in an enzyme superfamily. Mol Cell Endocrinol 1997 ; 128 : 179-94.

4. Buck J, Sinclair ML, Schapal L, Cann MJ, Levin LR. Cytosolic adenylyl cyclase defines a unique signaling molecule in mammals. Proc Natl Acad Sci USA 1999 ; 96 : 79-84.

\section{Pascale Borensztein}

Inserm U. 474, Maternité Port-Royal, 123, boulevard de Port-Royal, 75014 Paris, France. 